\title{
Antitumor effect of murine dendritic and tumor cells transduced with IL-2 gene
}

\author{
Justyna Wojas-Turek, Elżbieta Pajtasz-Piasecka, Joanna Rossowska, Egbert Piasecki, \\ Danuta Duś
}

\author{
Ludwik Hirszfeld Institute of Immunology and Experimental Therapy, \\ Polish Academy of Sciences, Wroclaw, Poland
}

\begin{abstract}
Interleukin (IL-) 2 acts on a number of types of immune cells promoting their effector functions. To replace systemic administration of recombinant form of this cytokine, various genetically modified cells have been used in different preclinical models for tumor growth inhibition. In this study, dendritic or tumor cells transduced with retroviral vector carrying IL-2 gene (JAWS II/IL-2, X63/IL-2, MC38/IL-2 cells) alone or combined with tumor antigenstimulated dendritic cells (JAWS II/TAg) were exploited to treat colon carcinoma MC38-bearing mice. After the peritumoral injection of vaccine cells, the tumor growth delay and the increase in the number of tumor infiltrating $\mathrm{CD}^{+}$and $\mathrm{CD} 8^{+} \mathrm{T}$ lymphocytes were noted. A considerable increase in $\mathrm{CD}^{+}$cell influx into tumor tissue was observed when JAWS II/IL-2 cells or JAWS II/TAg with syngeneic MC38/IL-2 cells were applied. The increase in intensity of $\mathrm{CD}^{+}$cell infiltration was associated with immune reaction triggered by the same combination of applied cells or JAWS II/TAg with allogeneic X63/IL-2 cells. The effect observed in vivo was accompanied by MC38/0 cell specific cytotoxic activity of spleen cells in vitro. Thus, the application of vaccines, including IL-2-secreting cells of various origins, was able to induce different antitumor responses polarized by exogenous IL-2 and the encountered tumor antigen. (Folia Histochemica et Cytobiologica 2012, Vol. 50, No. 3, 414-419)
\end{abstract}

Keys words: MC38 murine colon carcinoma, IL-2 gene transduction, dendritic or tumor cell vaccine

\section{Introduction}

Interleukin (IL-) 2 is a pleiotropic cytokine acting on a number of types of immune cells to promote their effector functions. Due to this, IL-2 has been used as a tool in numerous clinical trials to fight growing tumors. Because of serious side effects observed after the systemic administration of IL-2, a number of alternative forms of therapy replacing the recombinant cytokines with genetically modified tumors as well as immune cells have been put forward [1-3]. Many studies have demonstrated that vaccination with IL-2-secreting tumor cells can cause considerable tumor growth inhibition, and additionally induce immunological memory [3-8]. On the

Correspondence address: E. Pajtasz-Piasecka, Institute of Immunology and Experimental Therapy, Polish Academy of Sciences, Laboratory of Experimental Anticancer Therapy, R. Weigla Str. 12, 53-114 Wroclaw, Poland; tel.: + 48713371172 ext. 373;

e-mail: pajtasz@iitd.pan.wroc.pl other hand, results obtained in mouse models as well as initial clinical trials have demonstrated that genetically modified dendritic cells (DCs) can attend the induction of anti-tumor immune responses followed by tumor regression [8].

The aim of our study was to compare the effects of the application of IL-2-transduced cells in a model of colon carcinoma MC38. The main question was whether IL-2 transductants of various origins would be able to support the antitumor activity of tumor antigen (TAg)-stimulated DCs with the same efficiency. The cytokine carriers were represented by syngeneic tumor (MC38/IL-2) cells or allogeneic plasmacytoma (X63/IL-2) cells. Genetically modified DCs originating from the immature dendritic cell of JAWS II line represented immune cells.

The obtained results demonstrate that the vaccines including particular IL-2-secreting-cells, independently of their origin, induced similar tumor growth delay. However, the use of IL-2-secreting-cells in combination with TAg-stimulated DCs diversified their effect on the antitumor immune response. 


\section{Material and methods}

Mice. Female C57BL/6 mice, 8-10 weeks old, were obtained from Mossakowski Medical Research Center, Polish Academy of Sciences, Warsaw. All animal experiments were approved by the Local Ethics Committee.

Cell lines. Parental cells of the MC38/0 murine colon carcinoma line [9] and genetically modified cells were maintained in RPMI 1640 supplemented with 5\% fetal bovine serum (FBS). The C57BL/6 murine bone marrow-derived DCs of the JAWS II line (ATCC Cat. No. CRL-11904, USA), as well as cells modified with IL-2 gene carried by retroviral vector, were cultured in a culture medium containing $10 \% \mathrm{FBS}$ and $5 \mathrm{ng} / \mathrm{ml}$ GM-CSF (Cytogen). The X63/Ag8.653 murine plasmacytoma cells transduced with IL-2 gene (Institute of Immunology and Experimental Therapy) were maintained in culture medium supplemented with $10 \%$ FBS. JAWS II cells and cells of both murine tumor lines were transduced with retroviral vector pQN carrying murine IL-2 gene (JAWS II/IL-2, MC38/IL-2, $\mathrm{X} 63 / \mathrm{IL}-2$ ) according to the previously described protocol [10]. Transductants produced cytokine at a relatively constant level and were not tumorigenic (data not shown).

Therapeutic schedules. Mice were inoculated subcutaneously (s.c.) in the right flank with MC38/0 colon carcinoma cells $\left(1 \times 10^{6}\right.$ cells $/ 0.2 \mathrm{ml}$ in PBS per mouse $)$. On the $14^{\text {th }}$ day after tumor inoculation, mice with palpable nodules were vaccinated peritumorally (p.t.) with dendritic JAWS II/IL-2 or tumor (X63/IL-2, MC38/IL-2) cells alone or in combination with JAWS II cells stimulated in vitro with tumor antigens (JAWS II/TAg). The vaccine consisted of ca. $1 \times 10^{6}$ cells (when combined $-0.7 \times 10^{6}$ of JAWS II/TAg cells were mixed with $0.3 \times 10^{6}$ of transductants). The cells were applied once a week, for three consecutive weeks. Tumor diameters were measured every $2-3$ days. On the $7^{\text {th }}$ day after the last injection ( $35^{\text {th }}$ day of the experiment), some of the mice were sacrificed, and spleens as well as tumors were dissected.

Tumor sections and immunohistological analysis. Tumor tissue cryo-sections, 5-10 $\mu \mathrm{m}$ thick, were fixed in acetone and incubated for 2 hours with rat anti-CD4 or anti-CD8 $\mathrm{mAb}$ (BD Pharmingen) followed by goat anti-rat antibody conjugated with AlexaFluor488 ${ }^{\circledR}$ (Molecular Probes) and counterstained with PI. The tissue sections were analyzed using a BioRad MRC 1024 scanning confocal fluorescence microscope equipped with LaserSharp software. Cryosections from three distinct parts of tumor from each individual mouse were analyzed.

Cytotoxicity assay of activated spleen cells. Spleen cells were co-cultured with mitomycin $\mathrm{C}$ treated MC38/0 cells for 5 days. After this in vitro stimulation, spleen cell cytotoxicity was investigated using a LIVE/DEAD Cell-Mediated
Cytotoxicity Kit (Molecular Probes). The cytotoxic index was determined by the percentage of dead (PI-positive) among DiO MC38/0 target cells. The analyses were carried out using BD FACSCalibur apparatus with the CELLQuest software.

\section{Results/Discussion}

Our study analyzed the effect of the vaccines containing cells transduced with retroviral vector pQN carrying murine IL-2 gene on the induction of antitumor immunity. Transductants produced cytokine at the level range $60-120 \mathrm{LU} / \mathrm{ml}$. They were represented by nontumorigenic syngeneic tumor cells (MC38/IL-2 cells) and allogeneic plasmacytoma cells (X63/IL-2 cells) and JAWS II/IL-2 cells originating from an immature dendritic cell line (for detailed characteristics of these cells, see [10]). The analysis was conducted on mice bearing advanced murine colon carcinoma (MC38) treated with IL-2-producing cells and/or tumor antigen-stimulated dendritic (JAWS II/TAg) cells (Figure 1).

The obtained data revealed similar therapeutic effects of IL-2-producing tumor cell vaccines. The injections of MC38/IL-2 or X63/IL-2 cells elicited $\triangle \mathrm{TRV}$ (difference in the median time required for the tumor to reach a volume of $1 \mathrm{~cm}^{3}$ compared to the control group of mice) amounting to 5.5 or 6.5 days, and TGI (tumor growth inhibition) amounting to 53 or $55 \%$, respectively. The application of JAWS II/IL-2 cells resulted in stronger tumor growth delay (up to seven days for $\Delta \mathrm{TRV}$, and TGI of 56\%; see insert in Figure 1). An explanation of their stronger effect on tumor growth delay could be based on two activities - they could act in a complex manner as a source of IL-2 and, to some extent [see 10], as antigen presenting cells. This suggests that although both tumor and dendritic vaccine cells were applied in the same numbers, their therapeutic effects depended not only on their ability to produce IL-2 but also on their various origins.

We noted that the application of JAWS II/TAg cells caused tumor growth delay for only four days compared to the control group; this is three days less than vaccine containing the same number of JAWS II/IL-2 cells. Given this, we decided to replace the $30 \%$ of JAWS II/TAg cells by JAWS II/IL-2 cells as well as by MC38/IL-2 or X63/IL-2 cells. The application of JAWS II/TAg cells along with JAWS II/IL-2 did not elicit further tumor growth delay. Thus, JAWS II/IL-2 cells which exhibited only low ability to present TAg [10] were not able to balance the effect of the part of TAg-stimulated DCs and obviously were not able to augment the vaccine's effectiveness.

We determined which type of vaccine tumor cells would be able to trigger efficient immunity against advanced tumor - the syngeneic cells presenting the same TAg as growing tumor or the allogeneic cells 


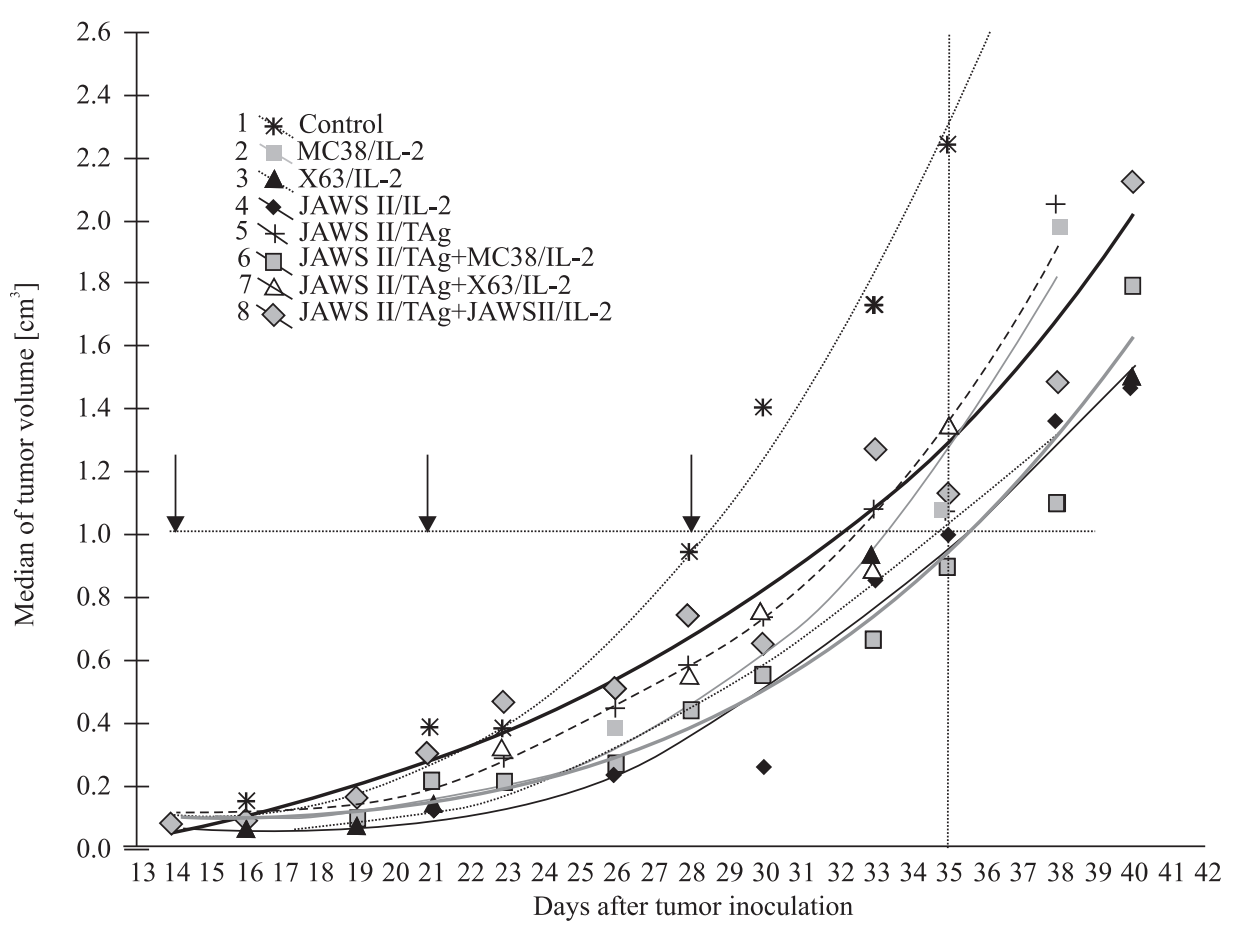

\begin{tabular}{l|cc} 
Vaccines & $\Delta \mathrm{TRV}^{*}($ days $)$ & $\mathrm{TGI} * *(\%)$ \\
\hline 1 Control & - & \\
2 MC38/IL-2 & 5.5 & 53 \\
3 X63/IL-2 & 6.5 & 55 \\
4 JAWS II/IL-2 & 7 & 56 \\
5 JAWS II/TAg & 4 & 52 \\
6 JAWS II/TAg+MC38/IL -2 & 7 & 61 \\
7 JAWS II/TAg+X63/IL -2 & 4 & 40 \\
8 JAWS II/TAg+JAWSII/IL -2 & 4 & 50
\end{tabular}

Figure 1. Differences in tumor growth delay as a result of cellular vaccine application. Specific pathogen-free condition (SPF)-female mice were injected with MC38/0 cells $\left(1.0 \times 10^{6}\right.$ cells $)$ on day 0 . Cell vaccines were applied in the vicinity of established tumors in three consecutive weeks, starting from the $14^{\text {th }}$ day. Transductants used as a component of vaccines produced $60-120 \mathrm{LU}$ of IL-2/ml $/ 0.5 \times 10^{6} \mathrm{cells} / 48 \mathrm{~h}$. The main figure includes the tumor growth delay graph presented as the median of the tumor volume (taken from five or six mice). Insert includes the median of the tumor volume (taken from five or six mice) calculated using the Kruskal-Wallis test on the $35^{\text {th }}$ day of the tumor growth. The table below presents: * $\triangle \mathrm{TRV}$ - difference in the median time required for the tumor to reach a volume of $1 \mathrm{~cm}^{3}$ compared to the control group of mice, days; **TGI - tumor growth inhibition, \%; calculated on the $35^{\text {th }}$ day (seven days after the third injection) according to the formula $100-(\mathrm{MVT} / \mathrm{MVC} \times 100)(\%)$ where MVT represents the median tumor volume in the treated mice and MVC the median tumor volume in the control mice

expressing antigens of distinctive $\mathrm{MHC}$ and representing the other type of malignancy. Based on the data described by others, we expected that the allogeneic IL-2-secreting transductants ought to be strong stimulators inducing response not only against themselves but also against the growing tumor. Meanwhile, the application of vaccines consisting of JAWS II/ /TAg + MC38/IL-2 cells caused the highest tumor delay $(\triangle \mathrm{TRV}-$ seven days and TGI $-61 \%)$, and after JAWS II/TAg + X63/IL-2 cell administration the weakest effect was observed ( $\triangle \mathrm{TRV}$ - four days, TGI - 40\%, Figure 1). Moreover, the use of JAWS II/ $/ \mathrm{TAg}+\mathrm{X} 63 / \mathrm{IL}-2$ cells shortened the survival time of mice (data not shown). Generally, it was revealed that the application of JAWS II/IL-2 cells as well as JAWS II/TAg + MC38/IL-2 cells affected stronger tumor growth delay than other groups (illustrated by $\Delta \mathrm{TRV}$ amounting to seven days and TGI - to $56 \%$ and $61 \%$, respectively), and the use of JAWS II/TAg + X63/IL-2 cells triggered adversarial immune reaction. Overall, tumor growth delays elicited by particular vaccines were noted, but the differences between them were not statistically significant. Nevertheless, our observations allow us to hypothesize that vaccines containing various type of cells elicited final results which may be mediated by different components of the immune system.

In many human cancers, the presence of $\mathrm{T}$ cells infiltrating into tumor tissue is believed to be a good prognostic marker for tumor regression [11]. The same relationship between the number of such lymphocytes and their antitumor effect can be observed 

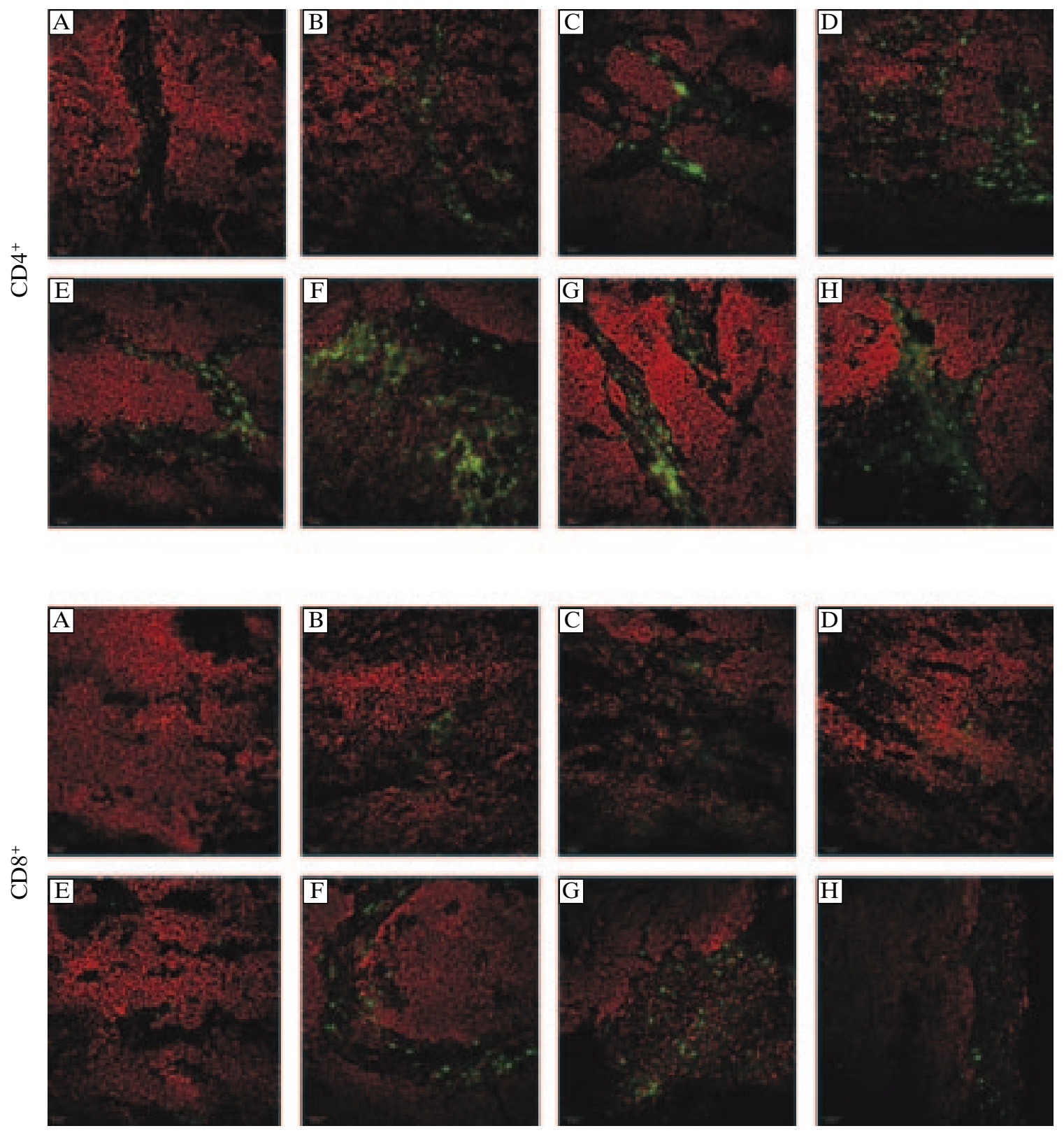

Figure 2. $\mathrm{CD}^{+}$or $\mathrm{CD}^{+}$cell infiltration into tumor tissues after cell vaccine treatment. Tumors were harvested on the $35^{\text {th }}$ day. Cryo-sections, fixed in acetone, were incubated with rat anti-mouse anti-CD4 or anti-CD8 antibodies followed by goat anti-rat antibody conjugated with AlexaFluor $488^{\circledR}$, and counterstained with PI. The analysis of tumor infiltrating lymphocytes was carried out using a scanning confocal fluorescence microscope. Magnification $\times 250$. A: Control, B: MC38/IL-2, C: X63/IL-2, D: JAWS II/IL-2, E: JAWS II/TAg, F: JAWS II/TAg + MC38/IL-2, G: JAWS II/TAg + X63/IL-2, H: JAWS II/TAg + JAWS II/IL-2

in experimental tumors. In our model, the application of dendritic and/or tumor cell vaccines induced changes of the tumor tissue structure which were associated with the increase in the number of both $\mathrm{CD}^{+}$ and $\mathrm{CD}^{+} \mathrm{T}$ cells. Generally, the influx of $\mathrm{CD} 4^{+}$cells was more intensive than that of $\mathrm{CD}^{+}$cells. However, the locations of these cells were various: $\mathrm{CD}^{+}$ cells were found in connective tissue in tumor nodules, whereas $\mathrm{CD} 8^{+}$cells were preferentially located between tumor cells. The intensity of lymphocyte in- flux depended on the type of vaccinated cells (Figure 2, Table 1).

The highest infiltration of tumor tissue by both $\mathrm{CD}^{+}$and $\mathrm{CD} 8^{+}$cells was observed after the injection of JAWS II/IL-2 cells. The application of IL-2-secreting tumor cells caused a moderate influx, higher than in the control group but comparable with that elicited by JAWS II/TAg cells. On the other hand, after the application of the combined cell vaccines, a differential lymphocyte influx was noted. An inten- 
Table 1. $\mathrm{CD}^{+}{ }^{+}$or $\mathrm{CD}^{+}$cell infiltration into tumor tissues after the cellular vaccine treatment

\begin{tabular}{|l|c|c|}
\hline Vaccine & \multicolumn{2}{|c|}{$\begin{array}{c}\text { Tumor infiltrating } \\
\text { lymphocytes*: }\end{array}$} \\
\hline & CD4 $^{+}$ & CD8 $^{+}$ \\
\hline Control & ++ & + \\
\hline MC38/IL-2 & +++ & ++ \\
\hline X63/IL-2 & +++ & ++ \\
\hline JAWS II/IL-2 & +++++ & +++ \\
\hline JAWS II/TAg & +++ & ++ \\
\hline JAWS II/TAg+MC38/IL-2 & +++++ & +++ \\
\hline JAWS II/TAg+X63/IL-2 & +++ & +++ \\
\hline JAWS II/TAg+ JAWS II/IL-2 & ++++ & ++ \\
\hline
\end{tabular}

*Intensity of CD4 and CD8 cell influx was estimated based on 4-5 representative tumor sections and is shown by the number of crosses, i.e. " + " = few cells, and " +++++ " = many cells

sive influx of $\mathrm{CD}^{+}$cells was observed after treatment with JAWS II/TAg + MC38/IL-2 cells, a moderate influx after JAWS II/TAg + JAWS II/IL-2 cells, and a small influx after JAWS II/TAg + X63/IL-2 cells. In turn, the combined cell vaccines containing transduced tumor cells caused a more intensive influx of CD8 ${ }^{+}$lymphocytes than JAWS II/TAg + JAWS II/IL-2 cells.

Our results demonstrate that the most intensive influx of both $\mathrm{CD}^{+}$and $\mathrm{CD}^{+}$cells into tumor tissue was observed when IL-2-producing DCs or, alternatively, TAg-stimulated DCs along with IL-2-secreting syngeneic tumor cells, were delivered into the tumor surroundings. Moreover, when both $\mathrm{CD}^{+}$and $\mathrm{CD}^{+}$lymphocytes appeared in tumor tissue at a high level, a positive correlation between the intensity of the lymphocyte influx and the inhibition of the tumor growth could be observed. As observed by Bos [12], $\mathrm{CD} 4^{+}$cells presented in tumor tissue could help in overcoming the immune tolerance by promoting the $\mathrm{CD}^{+}$cell recruitment and activation of their cytolytic function together with the enhancement of the effector and memory $\mathrm{CD} 8^{+}$cell survival. The application of JAWS II/TAg + X63/IL-2 cell combination caused an influx of $\mathrm{CD}^{+}$cells similar to the other combined vaccines. Nevertheless, they elicited an unexpected overall effect inducing a weaker inhibition of MC38 tumor growth (the lowest TGI) and shortening the survival time of mice (data not shown). Presumably, this was a consequence of the simultaneous presence, in the vicinity of a growing tumor, of the TAg-stimulated DCs and allogeneic tumor cells. This parallel reaction directed towards two types of tumor cells probably caused an arrest of tumor growth de-

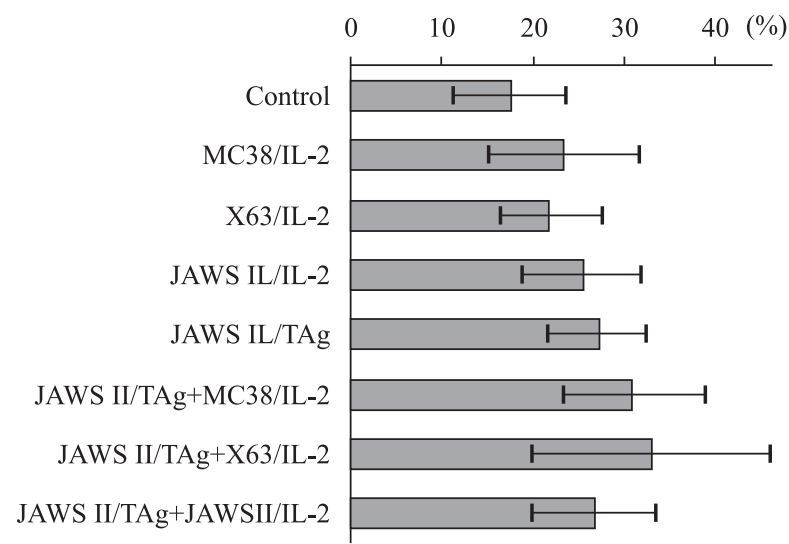

Figure 3. Cytotoxicity of restimulated spleen cells. Spleen cells were co-cultured with mitomycin C treated MC38/0 cells for five days. After this time (restimulation), splenocytes were co-cultured with target MC38 cells stained with DiO (MC38/DiO). Next, MC38/0-DiO cells were stained with PI and analyzed using flow cytometry. Cytotoxic index was determined by the percentage of dead (PI-positive) cells among MC38/0-DiO cells

lay. Thus, the application of allogeneic X63/IL-2 cells alone caused more effective tumor growth delay than the application of JAWS II/TAg + X63/IL-2 cell combination despite the level of CD8 ${ }^{+}$lymphocyte infiltration increasing after the latter vaccination. The application of vaccines of various origins seemed to be able to polarize the antitumor response by exogenous IL-2 and the encountered tumor antigen. This could result in a division of tumor infiltrating $\mathrm{CD}^{+}$ or $\mathrm{CD}^{+}$lymphocyte, down-regulated by tumor microenvironment.

To answer the question as to whether an increase in the number of infiltrating cells would be associated with the level of lymphoid cell cytotoxicity, the splenocytes obtained from the treated mice were in vitro co-cultivated with MC38/0 tumor cells. Cytolitic activity of splenocyte seemed to positively correlate with the level of $\mathrm{CD}^{+}$lymphocyte infiltration of tumor tissue (Figure 2). Furthermore, the application of IL-2-producing cells and the increase in the $\mathrm{CD}^{+}$ cell influx indicates the direct influence of IL-2 on the activity of these lymphocytes.

This drew our attention to the complex relationship between type of vaccine cells, cytokine produced by them, level of infiltration of immune cells into tumor tissue, and tumor ability to produce immunosuppressive cytokine such as TGF- $\beta$ (data not shown).

Hence, we hypothesize that differences in the delay of tumor growth are a consequence of various immune cell inductions resulting in the polarization of the final antitumor response. 


\section{Acknowledgements}

This work was supported by grants from the Polish Ministry of Science and Higher Education (No N N401 235334) and by the National Science Center of Poland (decision number DEC-2011/01/N/N24/ /01725).

\section{References}

1. Escobar A, López M, Serrano A et al. Dendritic cell immunizations alone or combined with low doses of interleukin-2 induce specific immune responses in melanoma patients. Clin Exp Immunol. 2005;142:555-568.

2. Ojima T, Iwahashi M, Nakamura $M$ et al. The boosting effect of co-transduction with cytokine genes on cancer vaccine therapy using genetically modified dendritic cells expressing tumor-associated antigen. Int J Oncol. 2005;28:947$-953$.

3. Melief CJ. Cancer immunotherapy by dendritic cells. Immunity. 2008;19;29:372-383.

4. Hoyer KK, Dooms H, Barron L, Abbas AK. Interleukin-2 in the development and control of inflammatory disease. Immunol Rev. 2008;226:19-28.

5. Devita VT, Hellman S, Rosenberg SA. Cancer: Principles and Practice of Oncology. 2005.
6. Kusnierczyk H, Pajtasz-Piasecka E, Koten JW, Bijleveld C, Krawczyk K, Den Otter W. Further development of local IL-2 therapy of cancer: multiple versus single IL-2 treatment of transplanted murine colon carcinoma. Cancer Immunol Immunother. 2004;53:445-452.

7. Podhajcer OL, Lopez MV, Mazzolini G. Cytokine gene transfer for cancer therapy. Cytokine Growth Factor Rev. 2007;18:183-194.

8. Pajtasz-Piasecka E, Kuśnierczyk H, Salwa J, Konarski L, Radzikowski C. Growth inhibition of transplantable tumors in mice by mIL-2-secreting murine plasmocytoma cells used alone or in combination with a cytostatic agent. Arch Immunol Ther Exp. 1995;43:281-292.

9. Pajtasz-Piasecka E, Szyda A, Rossowska J et al. Loss of tumorigenicity of murine colon carcinoma MC38/0 cell line after transduction with a retroviral vector carrying murine IL-12 genes. Folia Biol (Praha). 2004;50:7-14.

10. Rossowska J, Pajtasz-Piasecka E, Ryśnik O et al. Generation of antitumor response by IL-2-transduced JAWS II dendritic cells. Immunobiology. 2011;216;10:1074-1084.

11. Galon J, Costes A, Sanchez-Cabo F et al. Type, density, and location of immune cells within human colorectal tumors predict clinical outcome. Science. 2006;29;313:1960$-1964$.

12. Bos R, Sherman LA: CD4 ${ }^{+} \mathrm{T}$-cell help in the tumor milieu is required for recruitment and cytolytic function of $\mathrm{CD}^{+}$ T lymphocytes. Cancer Res. 2010;1;70:8368-8377.

Submitted: 15 December, 2011 Accepted after reviews: 22 February, 2012 